https://helda.helsinki.fi

\title{
Three-dimensional reconstruction of multiple particle acceleration regions during a coronal mass ejection
}

Morosan, D. E.

2020-03-09

Morosan , D E , Palmerio , E , Pomoell , J , Vainio , R , Palmroth , M \& Kilpua , E K J 2020 , '

Three-dimensional reconstruction of multiple particle acceleration regions during a coronal mass ejection ' , Astronomy \& Astrophysics , vol. 635 , A62 . https://doi.org/10.1051/0004-6361/201937133

http://hdl.handle.net/10138/320871

https://doi.org/10.1051/0004-6361/201937133

unspecified

publishedVersion

Downloaded from Helda, University of Helsinki institutional repository.

This is an electronic reprint of the original article.

This reprint may differ from the original in pagination and typographic detail.

Please cite the original version. 


\title{
Three-dimensional reconstruction of multiple particle acceleration regions during a coronal mass ejection ${ }^{\star}$
}

\author{
D. E. Morosan ${ }^{1}$, E. Palmerio ${ }^{1,2}$, J. Pomoell ${ }^{1}$, R. Vainio ${ }^{3}$, M. Palmroth ${ }^{1,4}$, and E. K. J. Kilpua ${ }^{1}$ \\ 1 Department of Physics, University of Helsinki, PO Box 64, 00014 Helsinki, Finland \\ e-mail: diana.morosan@helsinki.fi \\ 2 Space Sciences Laboratory, University of California-Berkeley, Berkeley, CA 94720, USA \\ 3 Department of Physics and Astronomy, University of Turku, 20014 Turku, Finland \\ ${ }^{4}$ Finnish Meteorological Institute, Space and Earth Observation Centre, PO Box 503, 00014 Helsinki, Finland \\ Received 18 November 2019 / Accepted 22 January 2020
}

\begin{abstract}
Context. Some of the most prominent sources for particle acceleration in our Solar System are large eruptions of magnetised plasma from the Sun called coronal mass ejections (CMEs). These accelerated particles can generate radio emission through various mechanisms.

Aims. CMEs are often accompanied by a variety of solar radio bursts with different shapes and characteristics in dynamic spectra. Radio bursts directly associated with CMEs often show movement in the direction of CME expansion. Here, we aim to determine the emission mechanism of multiple moving radio bursts that accompanied a flare and CME that took place on 14 June 2012.

Methods. We used radio imaging from the Nançay Radioheliograph, combined with observations from the Solar Dynamics Observatory and Solar Terrestrial Relations Observatory spacecraft, to analyse these moving radio bursts in order to determine their emission mechanism and three-dimensional (3D) location with respect to the expanding CME.

Results. In using a 3D representation of the particle acceleration locations in relation to the overlying coronal magnetic field and the CME propagation, for the first time, we provide evidence that these moving radio bursts originate near the CME flanks and that some are possible signatures of shock-accelerated electrons following the fast CME expansion in the low corona.

Conclusions. The moving radio bursts, as well as other stationary bursts observed during the eruption, occur simultaneously with a type IV continuum in dynamic spectra, which is not usually associated with emission at the CME flanks. Our results show that moving radio bursts that could traditionally be classified as moving type IVs can represent shock signatures associated with CME flanks or plasma emission inside the CME behind its flanks, which are closely related to the lateral expansion of the CME in the low corona. In addition, the acceleration of electrons generating this radio emission appears to be favoured at the CME flanks, where the CME encounters coronal streamers and open field regions.
\end{abstract}

Key words. Sun: corona - Sun: radio radiation - Sun: particle emission - Sun: coronal mass ejections (CMEs)

\section{Introduction}

Flares and coronal mass ejections (CMEs) from the Sun are the most powerful and spectacular explosions in the Solar System that are capable of releasing vast amounts of magnetic energy over a relatively short period of time. These phenomena are often associated with particle acceleration processes, including acceleration of electrons that can generate emission at radio wavelengths through various mechanisms.

The standard picture of CMEs predicts that they generally erupt when twisted or when sheared magnetic fields in the corona become unstable (Chen 2017; Green et al. 2018). As they propagate to higher coronal heights, CMEs are often seen in white-light imagery as a bright core and a dark cavity surrounded by a bright compression front (i.e. the classic three-part structure; Illing \& Hundhausen 1985). The cavity is believed to correspond to a magnetic helical structure, known as a "flux rope" (Dere et al. 1999; Vourlidas et al. 2013), which is an integral part of every CME regardless of its pre-eruptive configuration (Forbes 2000; Aulanier et al. 2010; Chen 2011).

\footnotetext{
Movies associated to Fig. 1 are available at https://www. aanda.org
}

When CMEs propagate faster than the characteristic speed of the ambient medium, they can drive shock waves that are capable of accelerating electrons to high energies. CME-driven shocks can be observed in white light as a fainter bright emission that surrounds the CME bubble (Vourlidas et al. 2003, 2013). Classically, the most obvious manifestations of shocks at radio wavelengths are a class of radio bursts called type II bursts, which are emitted by the plasma emission mechanism (Nelson \& Melrose 1985; Mann et al. 1996; Klassen et al. 2002). Type II radio bursts show emission sources that are closely associated with expanding CME-driven shocks in the corona (Zucca et al. 2018; Stewart et al. 1974). Fine-structured bursts called herringbones, which can accompany type II bursts or occur on their own (Holman \& Pesses 1983; Cairns \& Robinson 1987; Cane \& White 1989), represent signatures of individual electron beams accelerated by a shock, predominantly at the CME flanks (Carley et al. 2013; Morosan et al. 2019a).

CMEs can also be accompanied by continuum emission at decimetric and metric wavelengths, known as type IV bursts, that can have either stationary or moving sources (for a review, see Bastian et al. 1998). Moving type IV radio bursts, in particular, are commonly believed to be generated either by electrons trapped inside the CME flux rope that emit gyro-synchrotron 
radiation (Boischot \& Clavelier 1968; Dulk 1973) or electrons accelerated at a reconnecting current sheet that form at the wake of an erupting flux rope, generating radio bursts at the plasma frequency (Gary et al. 1985; Vršnak et al. 2003; Morosan et al. 2019b). In the former case, the radio source thus moves with the outward propagating CME and in the latter case the current sheet producing the type IV electrons also moves upwards in the corona as it is dragged by the rising CME. Moving type IV radio bursts, as well as type IIs and herringbones, can therefore be directly related to the outward propagation of CMEs in the solar corona. However, only type II radio bursts and herringbones have been directly related to CME shocks. So far, electron acceleration has only been observed at selective locations at the CME flanks in the low corona (Carley et al. 2013; Morosan et al. 2019a), despite the extended structure of the shock. The three-dimensional (3D) location of electron acceleration regions in relation to the propagating CME structure and surrounding corona is also not well known.

In this paper, we present observations of the radio emission associated with a CME that erupted on 14 June 2012. Using 3D modelling of the magnetic field environment and the CME structure, we show that multiple moving radio sources accompanying the CME represent plasma radiation near the CME flanks, where the CME is most likely to drive a shock. In Sect. 2, we give an overview of the observations and data analysis techniques used. In Sect. 3, we introduce the results of the moving radio bursts analysis, which are further discussed in Sect. 4. Finally, we present our conclusions in Sect. 5.

\section{Observations and data analysis}

On 14 June 2012, three moving radio sources were observed in images from the Nançay Radioheliograph (NRH; Kerdraon \& Delouis 1997) that appeared to follow the propagation direction of a CME accompanying a M1.9-class solar flare. This CME was well observed in remote-sensing observations by the Solar and Heliospheric Observatory (SOHO; Domingo et al. 1995) and Solar Dynamics Observatory (SDO; Pesnell et al. 2012) spacecraft that are located near Earth. The CME was also observed by the twin Solar Terrestrial Relations Observatory (STEREO; Kaiser et al. 2008) spacecraft orbiting the Sun close to $1 \mathrm{AU}$ and, at the time of this study, separated from Earth by $116^{\circ}$ and $117^{\circ}$, respectively. The onset and eruption of the 14 June 2012 CME from multi-wavelength imagery of the solar disc have been analysed extensively in several studies (James et al. 2017; Palmerio et al. 2017; Wang et al. 2019). The first observation of the CME in white-light coronagraph images was at 13:25 UT in the STEREO's inner coronagraph COR1, which is part of the Sun Earth Connection Coronal and Heliospheric Investigation (SECCHI; Howard et al. 2008) suite.

The NRH radio data ${ }^{1}$ were processed using the standard SolarSoft NRH package to produce calibrated radio images of the Sun. In the NRH images, we identified three radio sources moving away from the Sun in south-westerly and south-easterly directions and that are labelled 1, 2, and 3 in order of appearance in Figs. 1a and b. Multiple other stationary radio sources were also observed associated with the CME eruption (see e.g. the unlabelled radio sources in Fig. 1.) The bottom row in Fig. 1 shows the CME 12 min later in images from the Large Angle Spectrographic Coronagraph (LASCO; Brueckner et al. 1995) onboard SOHO. The CME propagated away from the Sun and towards the observer from Earth's perspective (Fig. 1c). The

\footnotetext{
https://rsdb.obs-nancay.fr
}

CME was relatively fast, with a plane-of-sky speed of $987 \mathrm{~km} \mathrm{~s}^{-1}$ as reported in the CDAW SOHO/LASCO CME Catalog ${ }^{2}$, and it expanded rapidly in longitude. A CME-driven shock is also visible in Fig. 1c (outlined by the black dots in all panels), which is identified as a sharp, fainter outer boundary surrounding the CME.

In dynamic spectra from the Nançay Decametric Array (NDA; Boischot et al. 1980) and the e-Callisto Birr spectrometer (Zucca et al. 2012), a period of continuum radio emission was observed which extended in frequency from a few hundred $\mathrm{MHz}$ to $20 \mathrm{MHz}$ and lasted for $\sim 2 \mathrm{~h}$, starting at 13:30 UT (Fig. 2a). This emission occurred approximately at the same time as the apex of the white-light front of the CME reached a height of $1.5 R_{\odot}$ (i.e. the inner edge of the STEREO/COR1 field of view). Such continuum radio emission is identified as a type IV radio burst, although it can consist of multiple components with varying emission mechanisms (i.e. plasma emission or gyro-synchrotron emission; Morosan et al. 2019b). Unfortunately, the resolution and sensitivity of the e-CALLISTO spectrometer, which observed the type IV burst at the same frequencies as NRH, are low. Therefore, it is hard to distinguish different components in the type IV continuum, however some fine-structured bursts are superimposed on this emission but they are not clearly identifiable (Fig. 2b). These fine structured bursts resemble herringbone bursts and, if herringbones or similar bursts are indeed present inside the type IV continuum, they would indicate the presence of electrons accelerated by a CME shock (Cairns \& Robinson 1987; Morosan et al. 2019a). Previous studies by Carley et al. (2013) have already identified herringbone bursts in the radio continuum following a type II burst, however in our case we do not see any clear signatures of shockaccelerated electrons in the dynamic spectra, such as a type IIs or herringbones.

\section{Results}

The moving radio bursts in Fig. 1 are mostly observed in images at 150 and $173 \mathrm{MHz}$ from the NRH (see Movies 1 and 2 accompanying this paper), except for Source 1 that is also sometimes prominent at $228 \mathrm{MHz}$. This indicates that the moving radio sources are narrow-band emission corresponding to a narrow frequency region of the time period observed in the dynamic spectrum in Fig. 2a, below $200 \mathrm{MHz}$. It is important to note that higher frequency emission $(>200 \mathrm{MHz})$, as well as other radio sources at 150 and $173 \mathrm{MHz}$, were also observed in NRH images. This emission can also be identified as type IV emission. However, this high-frequency radio emission seems to have no relation to the moving radio sources at 150 and $173 \mathrm{MHz}$, but it appears to correspond instead to stationary radio bursts located on top of the flaring active region during the CME expansion (see Figs. 1a and $\mathrm{b}$ and Movie 1 accompanying this paper). Here, we only focus on the moving radio sources, while the stationary radio sources will be investigated separately in a follow-up study. We therefore refer to the radio sources that show movement in Fig. 1 as moving radio bursts throughout this paper, as it is unclear if they are indeed part of the type IV continuum or if they are individual bursts superimposed on the type IV continuum.

The emission mechanism of the moving radio bursts can provide some insight into the origin of electron acceleration and their relation to the accompanying CME. Hence, the flux densities of the moving radio bursts are estimated for each of the

\footnotetext{
2 https://cdaw.gsfc.nasa.gov/CME_list/
} 
13:35:00 UT

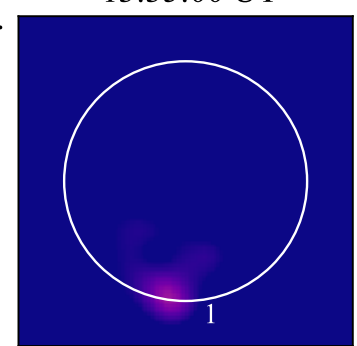

13:50:00 UT

b.

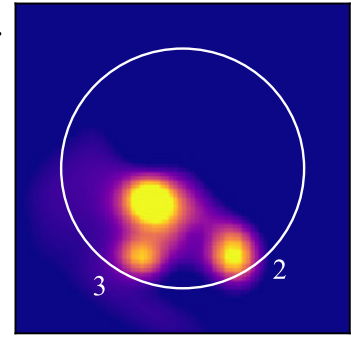

14:12:07 UT

c.

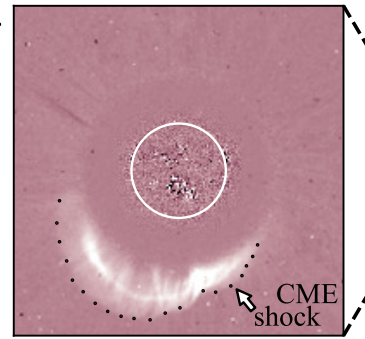

13:40:00 UT

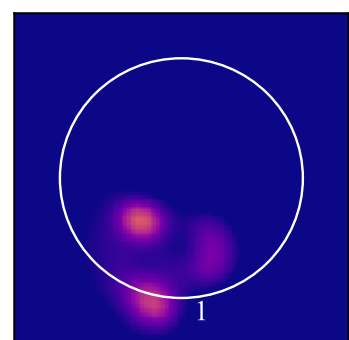

13:55:00 UT

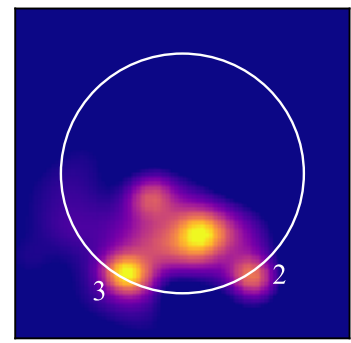

14:24:05 UT

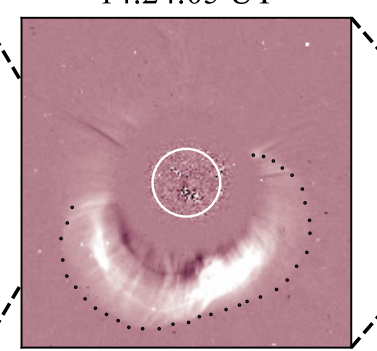

13:45:00 UT

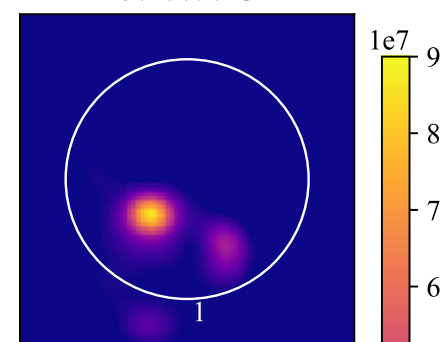

14:00:00 UT

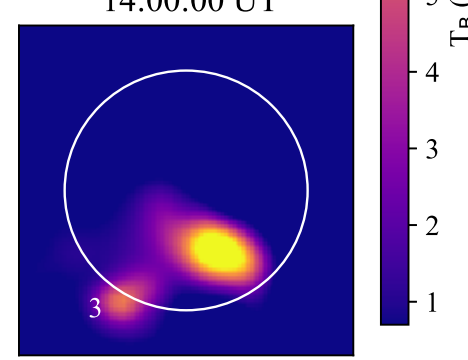

14:48:05 UT

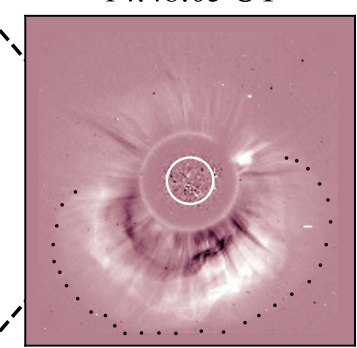

Fig. 1. Moving radio bursts associated with the expansion of the 14 June 2012 CME in the solar corona. The radio bursts are shown in panels $a$ and $b$ from 13:35 to 14:00 UT as observed by the NRH at $150 \mathrm{MHz}$. The white-light signature of the CME becomes visible at a later time (from 14:12 UT), when it expands to the higher corona as observed by the LASCO/C2 coronagraph. The white circles in the images in panel $c$ denote the visible solar limb. The white arrow points towards the CME shock, which is indicated by the fainter outline surrounding the CME. The outer edge of the white-light shock is denoted by the dotted line in panel $c$. A clear shock is observed in both southeasterly and south-westerly directions. The evolution of the radio bursts through time can be seen in Movies 1 and 2 available online. three sources observed. These flux densities are estimated inside a box covering the full extent and movement of the radio source and they include the pixels with levels $>20 \%$ of the maximum intensity levels in each box. We choose a treshold of $20 \%$ to include the full extent of the radio source and exclude quiet-Sun emission. The flux densities are estimated in solar flux units (sfu; where $1 \mathrm{sfu}=10^{-22} \mathrm{~W} \mathrm{~m}^{-2} \mathrm{~Hz}^{-1}$ ) for total intensity (Stokes $I$ ) and intensity of circularly polarised radiation (Stokes $V$ ) and are presented in Fig. 3. We note that there is an uncertainty in the absolute calibration of NRH data of $\sim 10-20 \%$ (see e.g. Chiuderi Drago et al. 1999; Carley et al. 2017). The emission is bursty and highly circularly polarised in the case of all moving radio sources. We also estimate the spectral index of the emission, $\alpha$, to help determine the possible emission mechanism. This is done by fitting a power-law function to NRH flux density spectra (plots of flux density vs. frequency), where the spectral index $\alpha$ is defined as the slope of a $\log -\log$ plot:

$\alpha=\frac{\mathrm{d} \log S}{\mathrm{~d} \log v}$,

where $S$ is the flux and $v$ is the observed frequency. The spectral index is fitted to two to three data points, since the moving radio bursts were only observed at 150 and $173 \mathrm{MHz}$ and rarely at $228 \mathrm{MHz}$. There were no moving radio bursts observed at frequencies above $228 \mathrm{MHz}$. The spectral index evolution through time is denoted by the $\times$-symbols in Fig. 3 .

All moving radio sources have a high degree of circular polarisation (see Fig. 3 and Movie 2 accompanying this paper). The first moving source (Source 1) consists of bursty emission that is $30-60 \%$ positively circularly polarised, based on our flux estimates and over the time interval shown. Source 1 is less bright compared to the later moving sources (Fig. 3a). The second and third moving sources (Sources 2 and 3) also consist of bursty emission that is up to $90 \%$ circularly polarised (Figs. $3 \mathrm{~b}$ and c), based on our flux estimates. We note, however, that the exact percentage of circular polarisation is difficult to estimate due to possible errors in the NRH absolute calibration procedures. However, the high signal-to-noise ratio in the Stokes $V$ images in the case of the moving radio sources indicates that there is indeed a strong component of circular polarisation. Sources 2 and 3 have an opposite sense of circular polarisation. Source 2 is positively polarised, similarly to Source 1 , while Source 3 is negatively polarised. This indicates different environments for the propagation of emitting electrons, such as access to differently orientated magnetic field lines (see Movie 2 accompanying this paper for the full evolution of the polarised moving sources). The spectral indices of all three moving sources are negative and steep, especially for Sources 2 and 3 that have spectral indices below -4 throughout their duration (see Fig. 3). In previous studies, the first moving burst (Source 1) was designated as emission originating from electrons trapped within the CME accelerated along magnetic field lines and emitting gyro-synchrotron radiation (James et al. 2017). However, the high degree of circular polarisation and steep spectral indices, especially for Sources 2 and 3 (Figs. 3b and c), are outside the range of known spectral indices for gyro-synchrotron emission. This suggests that the emission does not necessarily originate within the CME itself. Furthermore, the emission of all three moving sources is narrow-band and bursty in nature. These properties are all indicative of fundamental plasma emission (Robinson 1978; Benz \& Tarnstrom 1976). Thus, in the next sections, we assume that the three moving radio sources are emitted by the plasma emission mechanism. 


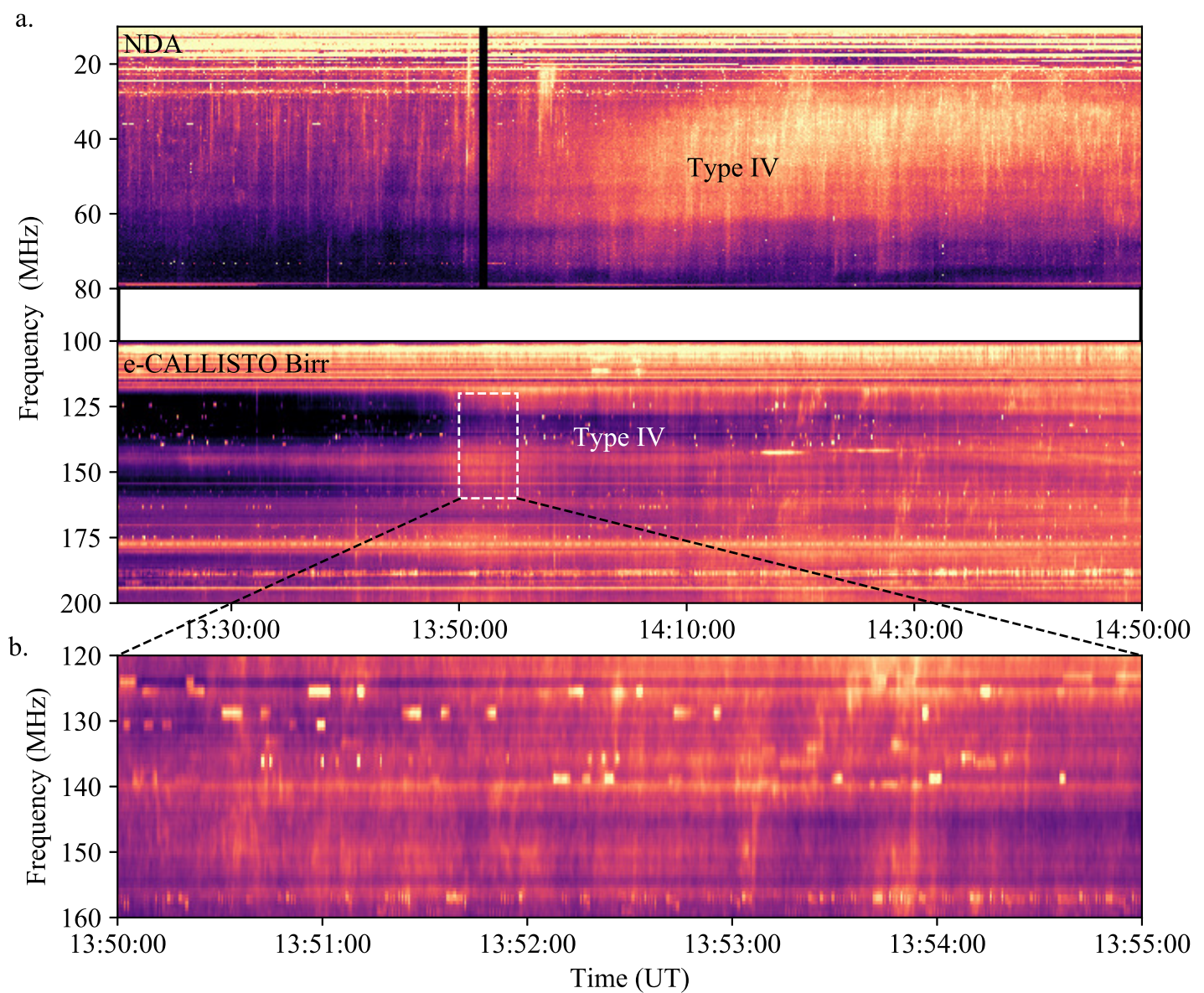

Fig. 2. Dynamic spectrum of the type IV radio burst observed on 14 June 2012. Panel a: dynamic spectra from the NDA (10-80 MHz) and the e-CALLISTO Birr spectrometer (100-200 MHz) showing a type IV continuum. Panel $b$ : zoom-in of the dynamic spectrum in $(a)$ showing some of the fine scale structures inside the type IV continuum at NRH frequencies.

\subsection{Radio source propagation from different perspectives}

Since our analysis in the previous section indicates that the moving radio sources are unlikely to be gyro-synchrotron radiation, here, we determine where the sources are located in relation to the CME body. The moving radio sources and CME originated within the visible solar disc (close to the central meridian), moving away from the Sun and towards the observer from Earth's perspective (Fig. 4). The radio centroids, coloured with progressing time, show the propagation direction of the radio bursts superposed on a plane-of-sky image at extreme ultraviolet (EUV) wavelengths from the Atmospheric Imaging Assembly (AIA; Lemen et al. 2012) onboard SDO (Fig. 4a). Source 1 and Source 3 move in a south-easterly direction, while Source 2 moves in a south-westerly direction. These propagation directions are coincident with the expansion of the associated $\mathrm{CME}$ and its shock wave outwards through the corona. However, from Earth's view, this plane-of-sky movement is affected by significant line-of-sight projection effects. Therefore, we construct a 3D perspective using observations from STEREO that were at ideal locations to provide side views of the eruption (see insert in Fig. 4a). The STEREO images in Figs. $4 \mathrm{~b}$ and $\mathrm{c}$ are composite images of the CME observed in the low corona at EUV wavelengths observed by the Extreme Ultraviolet Imager (EUVI) and white-light images from the COR1 coronagraph that observed the CME higher in the corona (the COR1 field of view extends between 1.5 and $4 R_{\odot}$ ). The CME expands radially and outwards from the solar limb in both STEREO-A and -B fields of view.
To determine the position of the radio sources in relation to the CME in the STEREO perspectives, we de-project the radio source centroids from their plane-of-sky view. Assuming that the radio bursts are emitted at the fundamental plasma frequency and do not originate from accelerated electrons inside the CME (see previous section), it is possible to determine their approximate height in the SDO perspective. The height of the radio bursts on the images away from the plane of sky is generally unknown, however electron density models of the solar corona can be used to provide the density stratification as a function of height, to de-project the radio source locations (Morosan et al. 2020). In the case of plasma emission, the electron density $\left(n_{\mathrm{e}}\right)$ can be related to the plasma frequency $\left(f_{\mathrm{p}}\right)$ using the following: $f_{\mathrm{p}} \sim 9000 \sqrt{n_{\mathrm{e}}}$. Therefore, for a certain radio burst emitted by the plasma emission mechanism, we can estimate its radial distance from the solar surface. We use the radially-symmetric electron density model of Newkirk (1961) to estimate the heights of the moving radio sources at a specific frequency. Assuming the radio bursts are emitted at the fundamental plasma frequency, the radial distance corresponding to the plasma frequencies of 150 and $173 \mathrm{MHz}$, respectively, is calculated using the four-fold Newkirk density model. This model also accounts for enhanced densities above active regions. This distance, combined with the plane-of-sky coordinates of the centroids, is then used to project the coordinates of the radio sources onto the STEREO planes and to represent them in 3D. There is however an uncertainty in estimating the radial distances of radio bursts using 

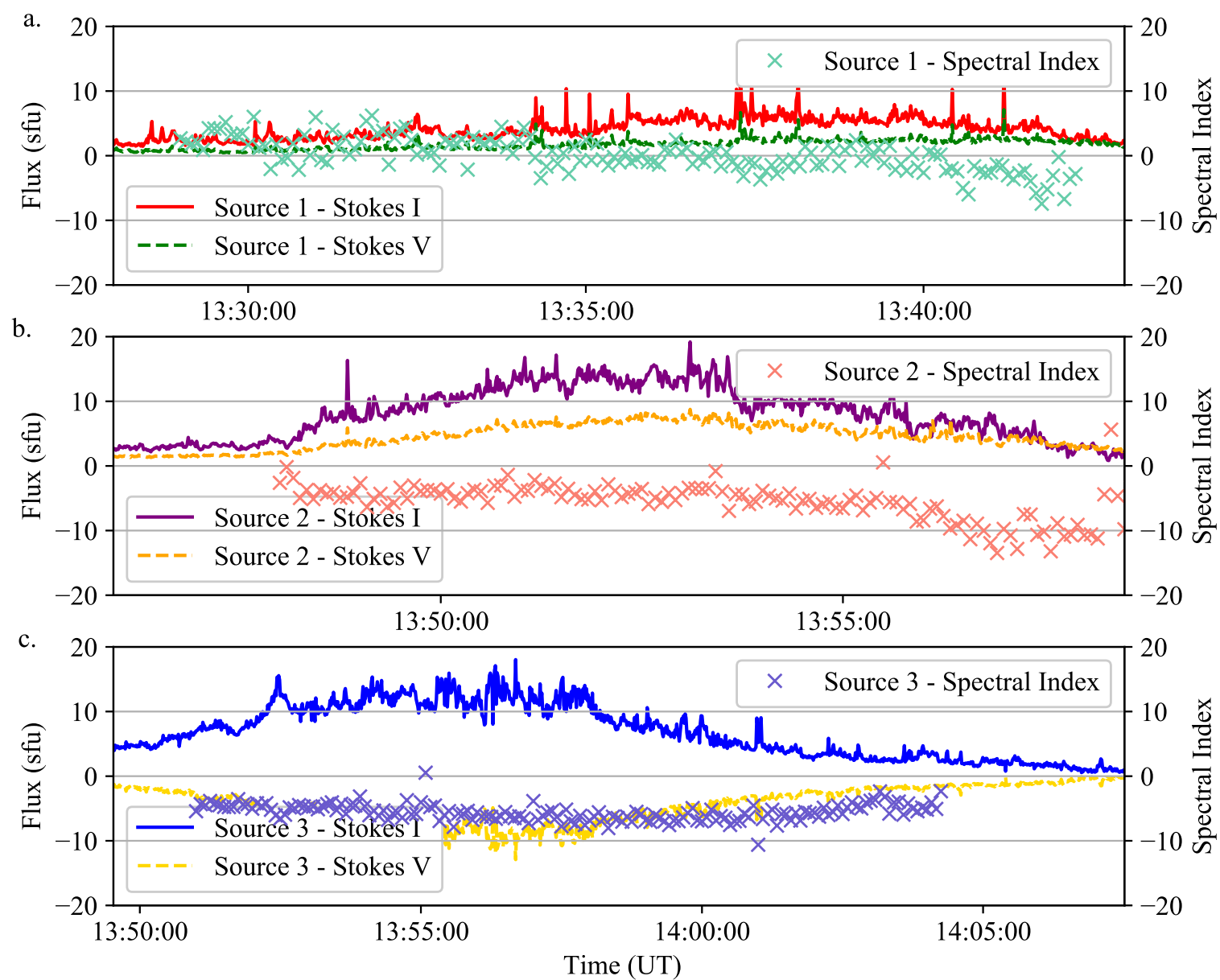

Fig. 3. Flux densities and spectral indices of the moving radio sources 1 (panel a), 2 (panel b), and 3 (panel c). The flux densities of the moving radio sources are estimated for both Stokes $I$ (solid lines) and Stokes $V$ (dashed lines) emission in each panel. The spectral indices were estimated by fitting a power-law function to the lower NRH frequencies, where the radio sources are clearly observed in images: $150-228 \mathrm{MHz}, 150-173 \mathrm{MHz}$, and $150-173 \mathrm{MHz}$, for Sources 1, 2, and 3, respectively.

density models. Firstly, the solar corona is variable and other density models can predict different distances. We therefore assigned an uncertainty of $\pm 0.3 R_{\odot}$ to the height estimates of the four-fold Newkirk model to reflect a range of possible heights of the radio sources. This was based on two other density models, one of the background solar corona (Saito et al. 1977) and one of the more active solar corona achieved through the sixfold Newkirk model. We also assign an uncertainty in estimating the plane-of-sky NRH centroids of $\pm 0.1 R_{\odot}$, which corresponds approximately to half of the extent of the full width at half maximum of the radio sources.

Using the 3D coordinates of the centroids, their approximate locations can be projected on the STEREO-B perspective for Sources 1 and 3 and on the STEREO-A perspective in the case of Source 2 (Fig. 5). The projections onto the STEREO planes reveal where the radio sources are located in relation to the CME and in which direction they move in relation to the CME expansion. All moving sources appear to be coincident with the southern CME flanks and move laterally in concert with the lateral expansion of the CME in the STEREO perspectives. In the case of Sources 1 and 2, the centroids are located outside the CME, in a region where the CME expands into a coronal streamer (labelled in Fig. 5). Source 3 appears to be located low inside the CME in the STEREO-B projection, however still closely related to the southern CME flank, and in 3D this source could be located outside, close to the CME legs. Despite the large uncertainty associated with such a de-projection technique, the used method would not affect the kinematics of the moving radio bursts in the STEREO perspectives, and these radio bursts would still follow the CME flank expansion to the south. We note that changing the density model used to de-project the centroids also does not affect the kinematics and position of radio sources at the CME flanks, the only effect being that the centroid position shifts either closer or farther away with respect to the flank.

At the southern CME flanks, where the moving radio bursts are located, the CME encounters a coronal streamer indicated in Fig. 5. The streamer appears to be perturbed by the passage of the CME, since its outline is changing in the running-difference images shown in Fig. 5. Since the streamer is located outside the CME, a wave driven by the CME expansion would most likely be able to perturb it. Densities inside coronal streamers are considered to be high, therefore the Alfvén speed inside streamers is low (Evans et al. 2008). Furthermore, the CME shows fast lateral expansion in the LASCO images in Fig. 1 and in the STEREO images in Fig. 5.

The CME speed was estimated by fitting an exponential height-time function, $h(t)$ from Gallagher et al. (2003), to planeof-sky EUVI and COR1 images from STEREO-A and -B. The lateral expansion speed of the CME was estimated from 13:20 to 14:10 UT by tracking the southern flank of the expanding CME in both STEREO-A and -B. Since the CME is located at the limb 
a. $\quad$ AIA $211 \AA 13: 45$ UT
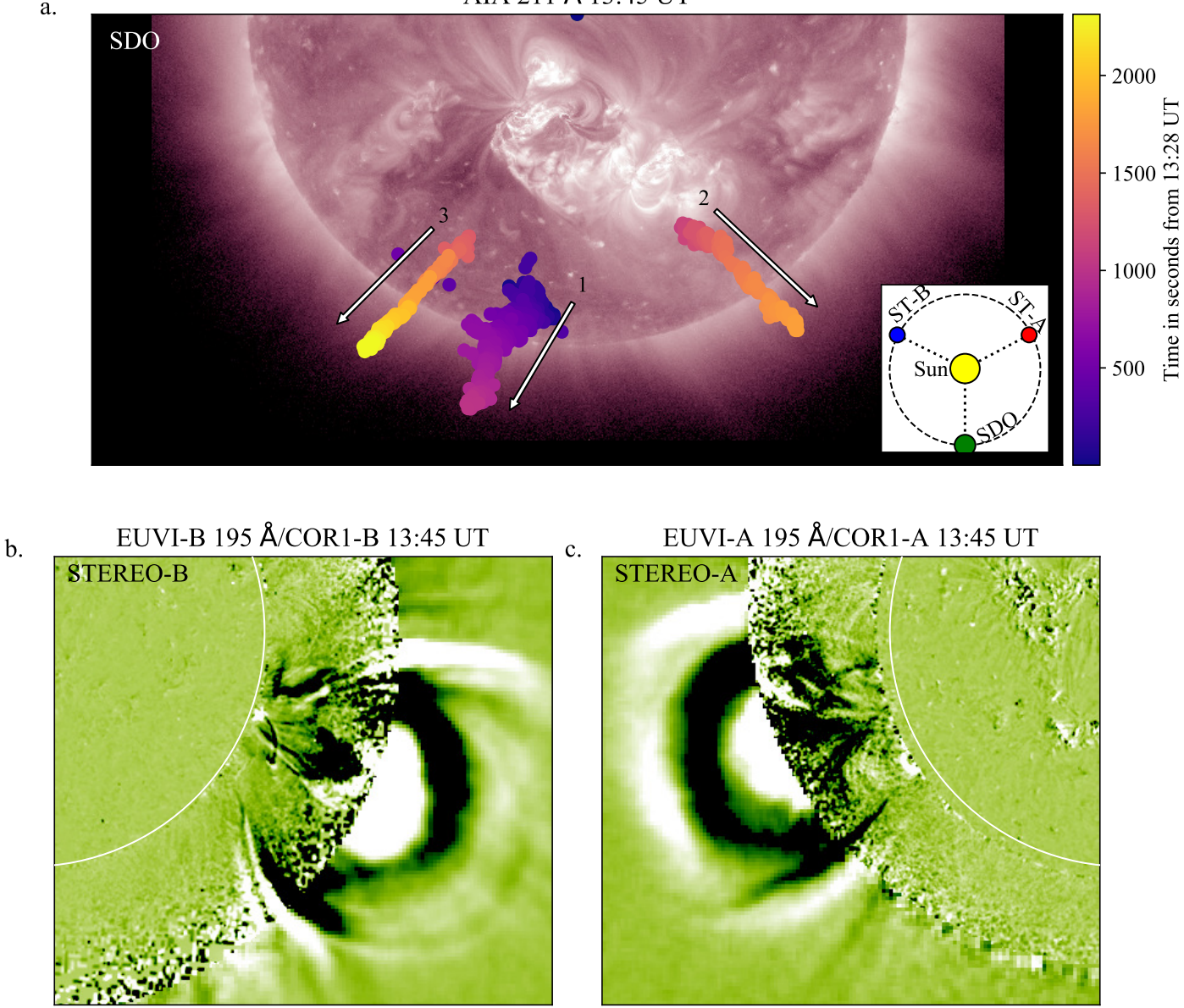

Fig. 4. Centroids of moving radio sources through time and associated solar activity visible from three perspectives. Panel $a$ : centroids of the three moving sources identified in Fig. 1, overlaid on an SDO/AIA $211 \AA$ image of the Sun at 13:45 UT. The centroids are colour-coded through time from 13:28 until 14:06 UT. In the AIA image, the source active region appears bright as a result of the sudden energy release of the M-class flare, however the accompanying CME is not visible. Panel $b$ : solar activity as viewed from the STEREO-B perspective that captures a side view of the flare and associated CME from the eastern side with respect to the SDO field of view at 13:45 UT. Panel $c$ : solar activity as viewed from the STEREO-A perspective that captures a side view of the flare and associated CME from the western side with respect to the SDO field of view at 13:45 UT. The EUV images at $195 \AA$ from EUVI are combined with coronograph images from the COR1 cameras onboard both STEREO-A and -B. The locations of SDO, STEREO-A, and STEREO-B in the ecliptic plane relative to the Sun can be seen in the insert in panel $a$.

in STEREO-A and -B, we expect an accurate estimate of the lateral expansion of the CME from this perspective. The heighttime function has the following form:

$h(t)=h_{0}+v_{0} t+a_{0} \tau^{2} \exp (t / \tau)$,

where $h_{0}, v_{0}$, and $a_{0}$ are the initial height, velocity and, acceleration, respectively. The function from Gallagher et al. (2003) fitted to the CME expansion contains an exponentially varying acceleration of the form $a_{0} \exp (t / \tau)$. The CME was found to expand rapidly towards the southern pole, reaching a speed of $>1000 \mathrm{~km} \mathrm{~s}^{-1}$ at 14:10 UT (Fig. 6a). This speed is larger than Alfvén speed values predicted inside coronal streamers of up to $\sim 400 \mathrm{~km} \mathrm{~s}^{-1}$ (Evans et al. 2008). The wave perturbing the streamer could in fact be a shock wave driven by the CME (Chen et al. 2010; Feng et al. 2012), especially after 13:55 UT when the CME speed is $>500 \mathrm{~km} \mathrm{~s}^{-1}$ (during the onset of Sources 2 and 3). A white-light enhancement indicating a shock is indeed observed surrounding the $\mathrm{CME}$ in LASCO/C2 images at a later time (Fig. 1c).

More evidence of a low-coronal shock is provided by the presence of a faint coronal or EUV wave (Fig. 6b). Running- difference images from SDO/AIA at $211 \AA$ show a faint propagating structure between 13:50 and 14:00 UT, which is propagating through the western hemisphere. This structure resembles a faint EUV wave, which represents a disturbance of the lowcoronal plasma caused by the outward expansion of the CME (Long et al. 2008). The shock wave driven by the CME in the low corona is believed to manifest itself as the EUV wave across the solar disc (Aschwanden 2009; Downs et al. 2012), therefore CME shocks, EUV waves, and particle acceleration are closely related (Kozarev et al. 2011; Grechnev et al. 2011).

\section{2. $3 D$ reconstruction of the electron acceleration locations}

The three perspectives from SDO-SOHO and the two STEREO spacecraft allow us to reconstruct the $3 \mathrm{D}$ structure of the CME evolving through the solar corona. Namely, we use the graduated cylindrical shell (GCS; Thernisien et al. 2006, 2009) model, which consists of a parameterised croissant-shaped grid, to manually trace the CME extent through time in the different planes of sky. The 3D reconstruction of the CME is then performed by overlaying the parametrised grid onto the three planes of sky (SOHO, STEREO-A, and STEREO-B) to reproduce the 
$13: 30$ UT

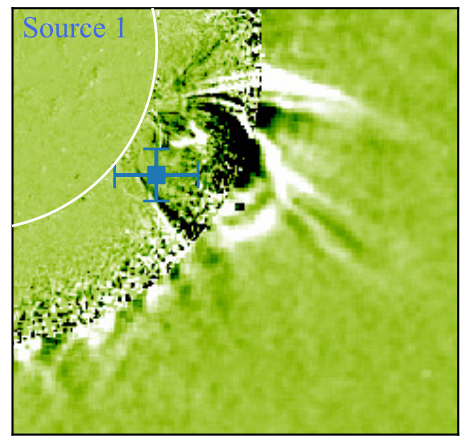

$13: 45$ UT

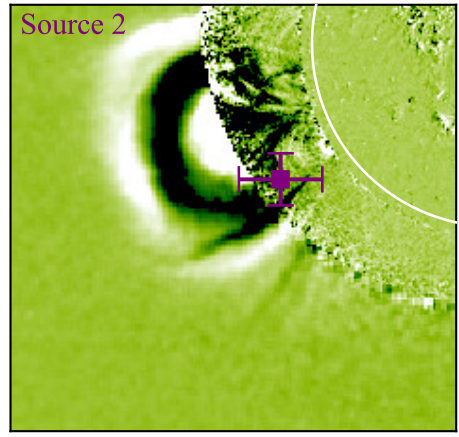

13:50 UT

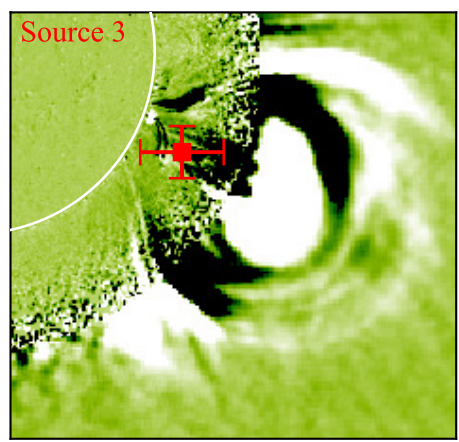

$13: 35$ UT

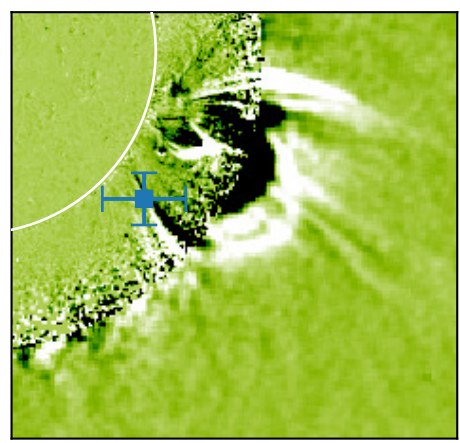

$13: 50 \mathrm{UT}$

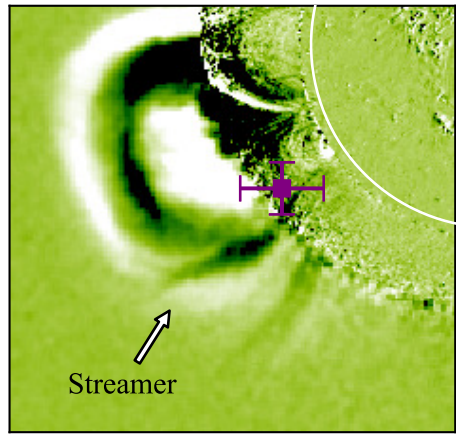

$13: 55$ UT

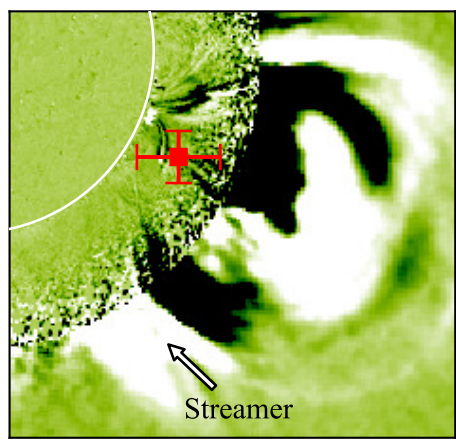

$13: 40 \mathrm{UT}$

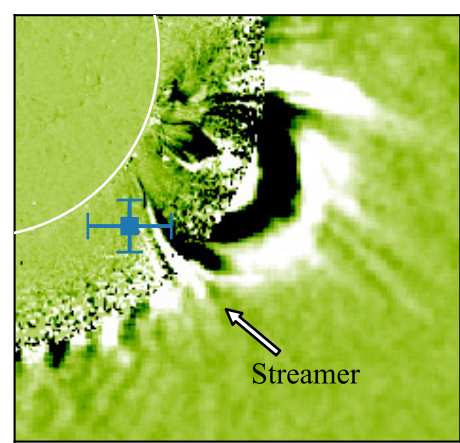

13:55 UT

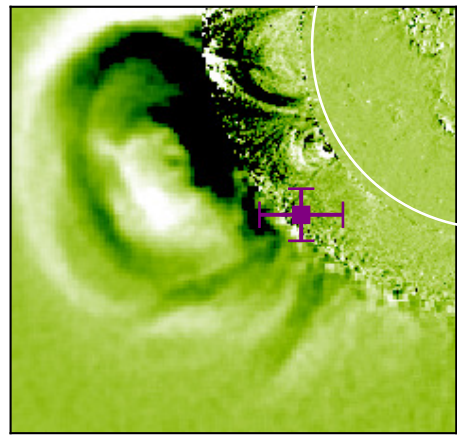

14:00 UT

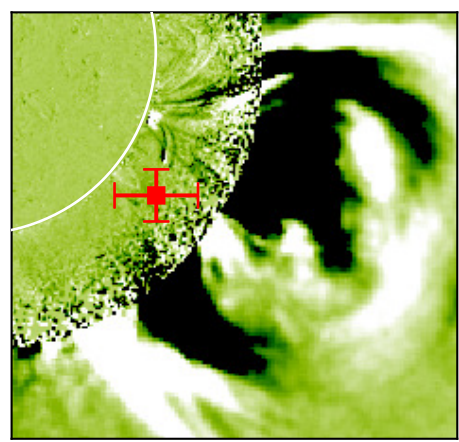

Fig. 5. Centroids of the moving radio sources projected onto the STEREO-A and -B perspectives over time. The STEREO images in each panel are composed of EUV images at $195 \AA$ from EUVI and coronagraph images from the COR-1 instruments onboard both STEREO-A and -B. Top and bottom panels: STEREO-B perspective, where the first and third moving sources can be seen. Middle panel: STEREO-A perspective, where the second moving source can be observed. The radio centroids coincide with the southern flank of the CME moving further southwards in each panel.

observed features at its best. We perform reconstructions from 13:40 to 14:10 UT (STEREO time) at five-minute intervals. Since the CME first appeared in the LASCO/C2 field of view at 14:12 UT, we perform reconstructions using only the COR1-A and -B viewpoints from 13:40 to 14:05 UT. We note that the fit to the CME bubble includes part of the nearest deflected streamer at the southern flank (visible in STEREO-A images in the middle panels of Fig. 5), as this is indicative of a shock wave perturbing the environment around the CME.

The reconstructed 3D CME bubble (wireframe consisting of magenta dots) is shown at three different times from top to bottom $(13: 40,13: 50$, and, 14:00 UT) and from the three perspectives from left to right (STEREO-B, SDO, and, STEREO-A) in Fig. 7. The middle panels of Fig. 7 show that the CME expanded very rapidly laterally in the plane of sky from Earth's view within a $30 \mathrm{~min}$ period. The speed of the CME southern flank from STEREO-B observations is also high, up to $1000 \mathrm{~km} \mathrm{~s}^{-1}$ before 14:10 UT (as determined in the previous section). The radio source contours (blue contours at $150 \mathrm{MHz}$ and orange contours at $173 \mathrm{MHz}$ ) are overlaid on the SDO images in the middle panels of Fig. 7. The contours of the first moving source at 13:40 UT (at $30 \%$ and $70 \%$ of the maximum intensity level) are located slightly outside the CME bubble. The radio contours of the second and third moving radio bursts appear to be located inside the CME bubble in the SDO viewpoint in Fig. 7. However, these radio sources can in fact be located behind the expanded CME bubble based on the centroid locations in Fig. 5 and on either side of the CME flanks. In the STEREO-A and -B images in Fig. 5, the radio centroids are indeed located at the CME flanks, which correspond to locations on either side of the CME legs in the SDO plane.

The CME reconstruction can be used to visualise the eruption and associated accelerated particles in 3D. The panels of Fig. 8 show the CME bubble and the coronal magnetic field, together with the location of the radio source centroids in 3D. In each of the panels in Fig. 8, the orange mesh represents the shape of the CME bubble in 3D and the extrapolated magnetic field lines around the $\mathrm{CME}$ are plotted as red and blue lines for 


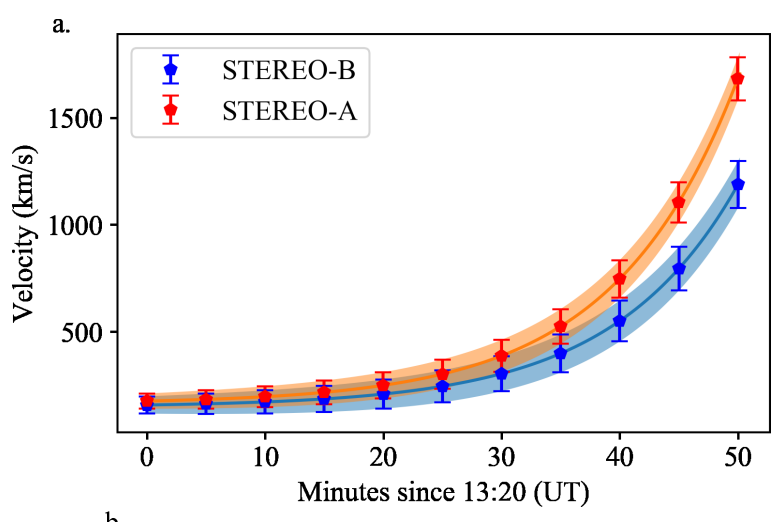

b.

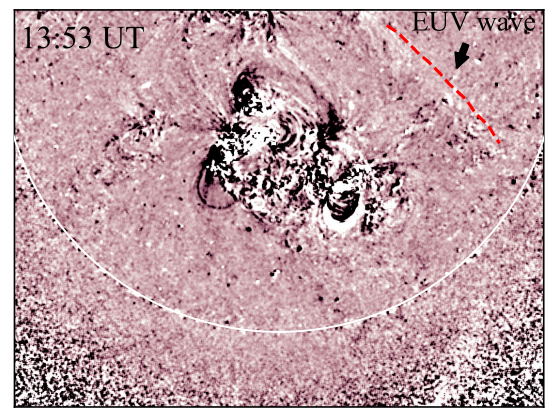

Fig. 6. Panel $a$ : lateral CME speed along the southern flank as observed in STEREO-A and -B. The CME speed data points were extracted from the composite EUVI and COR1 images from 13:20 through 14:10 UT, at 5 min intervals. The CME reaches a speed of $>1000 \mathrm{~km} \mathrm{~s}^{-1}$ at 14:10 UT in both STEREO-A and -B. Panel $b$ : faint EUV wave associated with the CME eruption, observed at the time of Sources 2 and 3 . The EUV wave was only observed on the western hemisphere. The fast CME lateral speed after 13:55 UT $\left(>500 \mathrm{~km} \mathrm{~s}^{-1}\right)$ and the presence of an EUV wave around the same time are strong indicators of a propagating shock wave surrounding the CME during the occurrence of Sources 2 and 3 .

the open field and grey lines for the closed field. Source 1 at 13:40 UT (blue sphere in the top row) is clearly located outside the CME at the southern flank. Source 2 (blue sphere in the bottom row) and Source 3 (red sphere in the bottom row) at 13:55 UT are located on either side of the CME western and eastern flanks, respectively. Based on the $3 \mathrm{D}$ reconstruction, the moving radio bursts observed in the plane of sky (the LASCO and SDO planes) represent the electromagnetic radiation produced at the CME flanks, most likely by shock-accelerated electrons given the fast speed of the CME expansion. These electrons are accelerated behind the overlying CME bubble seen in the SDO perspective, but our reconstruction shows that they can be located outside the CME bubble in other perspectives.

\subsection{Magnetic environment of the accelerated electrons}

Another noteworthy finding of the moving radio bursts studied here is their sense of circular polarisation. Langmuir (plasma) waves are generated by accelerated electron beams propagating through a plasma. For Langmuir waves travelling parallel or antiparallel to the magnetic field, the polarisation is in the sense of the ordinary (o-) mode (Dulk et al. 1976; Dulk \& Suzuki 1980), which is the only mode that can propagate through a coronal plasma to produce fundamental plasma emission (Melrose 2009). Fundamental radiation is then expected to be up to $100 \%$ polarised (Melrose \& Sy 1972). The o-mode resides on the same dispersion surface as the 1-mode (the left-handed cir- cularly polarised mode) and, hence, o-mode electromagnetic waves become circularly polarised following the refraction of the waves to the field-aligned direction (Oakes et al. 1979).

The polarisation of the moving radio bursts in this study, emitted at the fundamental plasma frequency, is most likely also in the sense of the o-mode. In particular, Sources 2 and 3 have a high degree of circular polarisation of up to $90 \%$ and, at the same time, an opposite (left and right, respectively) sense of circular polarisation from the viewpoint of the observer, which remains consistent throughout their duration. Extrapolations of the photospheric magnetic field (Schrijver \& De Rosa 2003) show that the CME is surrounded on either side by open-field regions of opposite polarity, in the plane of sky (Fig. 9). Since Sources 2 and 3 are also located on either side of the CME in the plane of sky, they are emitted in these open field regions of opposite polarity, where red field lines denote magnetic field from negative polarity regions and blue field lines denote magnetic field from positive polarity regions (Fig. 9). The magnetic field is oppositely oriented in the case of these two radio sources, therefore, their sense of polarisation is also opposite from the point of view of the observer.

Opposite senses of circular polarisation at low radio frequencies have been reported before by Kai (1970) in the case of type I noise storms (stationary emission accompanying active regions) and stationary type IV radio bursts, both believed to originate due to electrons accelerated inside small-scale coronal loops. Moving type IV radio bursts with opposite senses of circular polarisation have also been previously observed by Stewart (1971). However, these radio sources initially had a lower degree of circular polarisation (30\%), and one of the moving sources lasted for over an hour, most likely representing synchrotron radiation from mildly relativistic electrons, as concluded in Stewart (1971). One of the radio sources observed by Stewart (1971) became up to $100 \%$ circularly polarised before it faded, while Smerd \& Dulk (1971) also presented previous observations of moving radio sources with a very high degree of circular polarisation, similar to our observations. A high degree of circular polarisation $(>50 \%)$ has also been identified in early observations of herringbones (Suzuki et al. 1980). Similar moving radio bursts have also been observed by Smerd \& Dulk (1971), however in the case of opposite polarity type IVs an expanding arch structure was identified, believed to be generated by synchrotron electrons. More recently, opposite senses of circular polarisation have also been observed in the case of opposite-polarity neighbouring coronal holes (McCauley et al. 2019). Similarly to our findings, Kai (1978) also concluded that the polarity of magnetic fields at the type IV emission sites is similar to the polarity of magnetic fields at the photosphere based on a statistical study of 30 moving type IV bursts. Kai (1978) showed that left-handed polarised bursts are predominantly associated with positive polarity fields and right-handed bursts are the opposite, as in Fig. 9.

\subsection{Propagation effects on the $3 D$ position of radio sources}

Radio emission produced close to the plasma frequency level can be affected by propagation effects (Robinson 1983; Poquerusse et al. 1988; McCauley et al. 2018). In our case, the radio sources emitted at the fundamental plasma frequency are therefore emitted close to the plasma level and are likely to be impacted by propagation effects such as refraction and scattering by density inhomogeneities in the corona. These effects can shift the true position of the observed radio source (Robinson 1983), often resulting in radio sources at higher altitudes than the predicted plasma level (Poquerusse et al. 1988; McCauley et al. 2018). However, McCauley et al. (2018) argued that propagation 


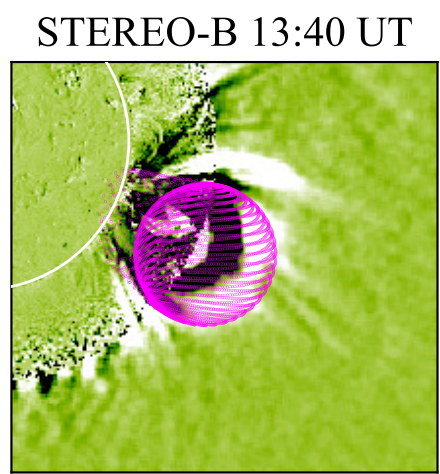

13:50 UT

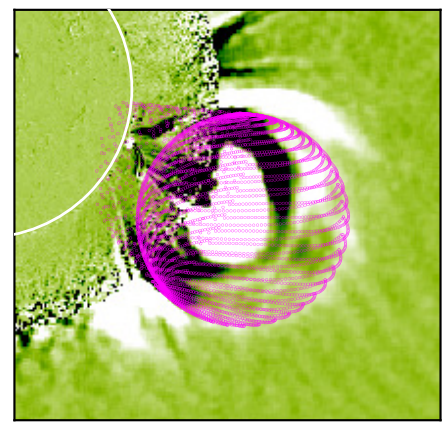

14:00 UT

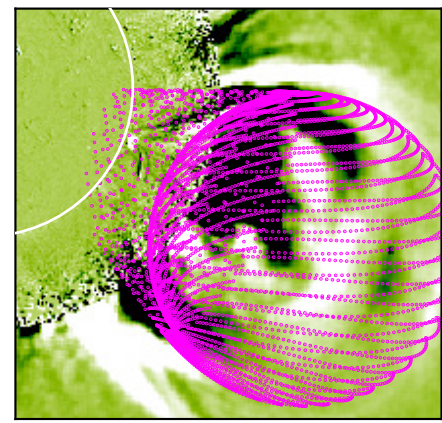

SDO 13:40 UT

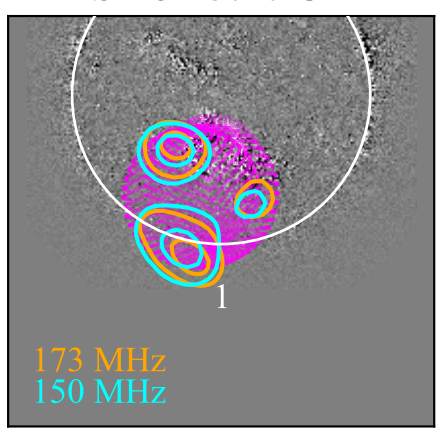

13:50 UT

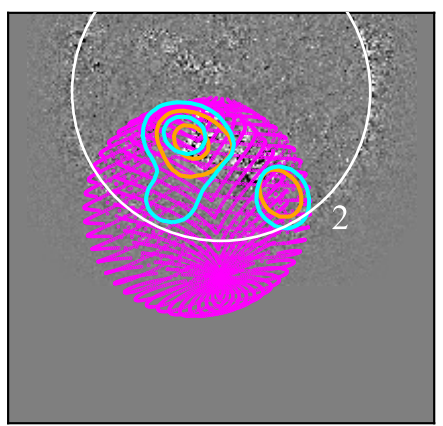

14:00 UT

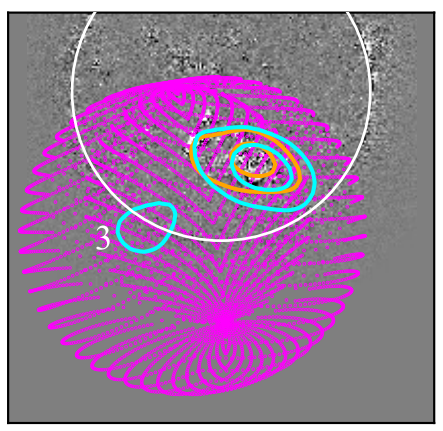

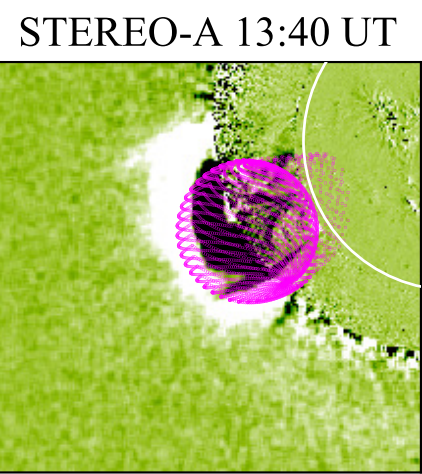

13:50 UT

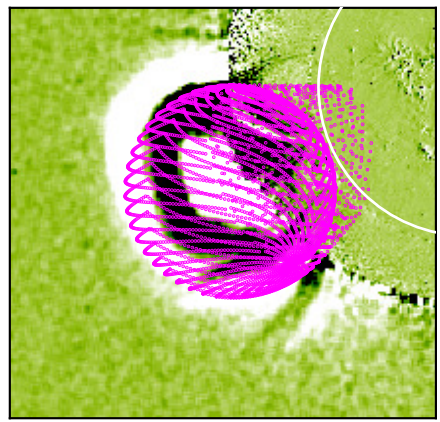

14:00 UT

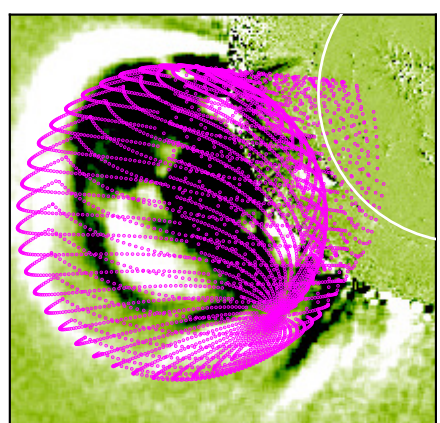

Fig. 7. 3D fitting of the CME bubble from three perspectives. The perspectives are images from STEREO-B, SDO, and STEREO-A from left to right, at three different times from top to bottom (13:40, 13:50, and 14:00 UT). The perspectives show the expansion of the CME fitted with the GCS model applied on the CME bubble (magenta dots). The radio contours at two frequencies (150 and $173 \mathrm{MHz}$ ) are overlaid in the middle panels on the SDO images.

effects only partially affect the position of the radio emission observed and that it is possible that the emitting electrons travel through over dense structures.

In our study, we used a four-fold Newkirk model to account for higher heights where low-frequency radio emission is observed. If propagation effects are indeed significant, our density model may predict too high densities to accurately reconstruct the radio emission in $3 \mathrm{D}$. However, the uncertainties in the radio source centroids in Fig. 5 were determined based on the positions predicted by a background density model of the solar corona on the lower end. A background model predicts that the source positions are closer to the CME and shifted in the direction of the CME core. These locations would place Source 2 completely inside the reconstructed CME shell, while Source 3 is partially inside the reconstructed shell within this uncertainty. Source 1 remains unaffected within this uncertainty. Therefore, if propagation effects are significant or the radial density model used is not adequate, there is a possibility that Sources 2 and 3 represent plasma radiation from inside the CME. We note, however, that this would have no effect on the kinematics of the moving radio bursts. All of the radio sources would still be closely related to the CME lateral expansion in the low corona, closely following the propagation of the CME flanks.

\section{Discussion}

We observed three moving radio sources with a high degree of circular polarisation that are best interpreted as plasma radiation. The nature and origin of the radio sources are interpreted as follows:

Source 1 occurs during the early stages of the CME eruption. In plane-of-sky observations, Source 1 appears to be located inside the apex of the CME, however in $3 \mathrm{D}$ reconstructions, the radio source is located significantly outside of the CME at its southern flank. There is however a discrepancy between the available orientation of open field and the sense of circular polarisation of Source 1. Source 1 has the same sense of circular polarisation as Source 2, however it occurs at a similar location as Source 3, where the orientation of the open field is negative. It is possible that we need to revise our interpretation of Source 1 as plasma emission outside the CME. Source 1 is unlikely to be related to a CME flank shock, as the CME may not be fast 

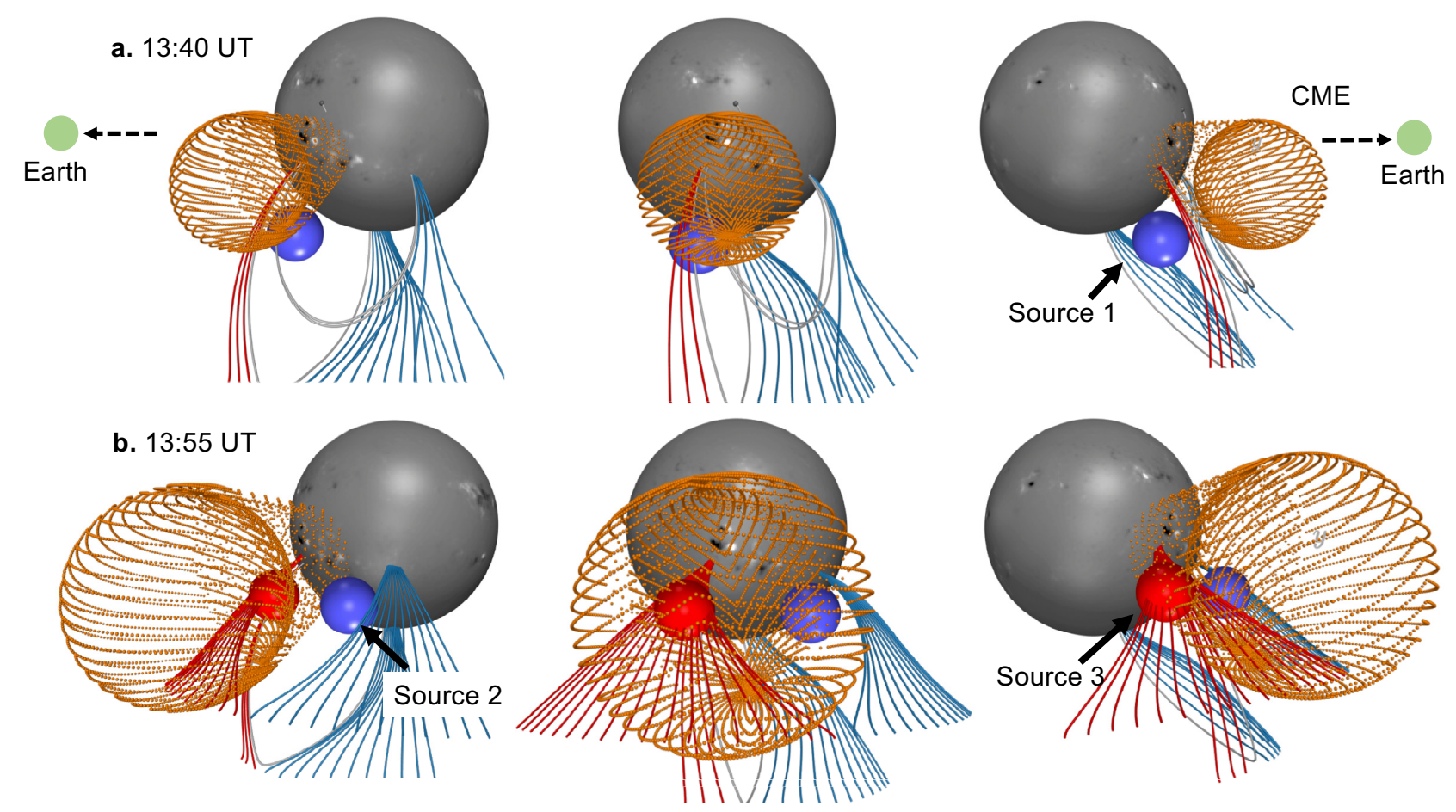

Fig. 8. 3D model of the CME bubble and electron acceleration locations. Each panel in this figure shows a different perspective similar to that of STEREO-A (left), plane-of-sky such as the SDO plane (middle), and STEREO-B (right). The magnetic field polarity (white for positive and black for negative) from photospheric magnetograms is overlaid on top of the surface of the solar sphere. Panel a: reconstruction of the CME eruption in the solar corona at 13:40 UT during the occurrence of Source 1. The orange mesh represents the CME bubble and the extrapolated magnetic field lines around the CME are plotted as red (negative polarity) and blue (positive polarity) lines for the open field and grey lines for the closed field. Source 1 (blue sphere) is located outside the CME. Panel $b$ : reconstruction of the CME eruption in the solar corona at 13:55 UT during the occurrence of Sources 2 and 3, which are located on either side of the CME. These radio sources are also located in open field regions surrounding the CME.

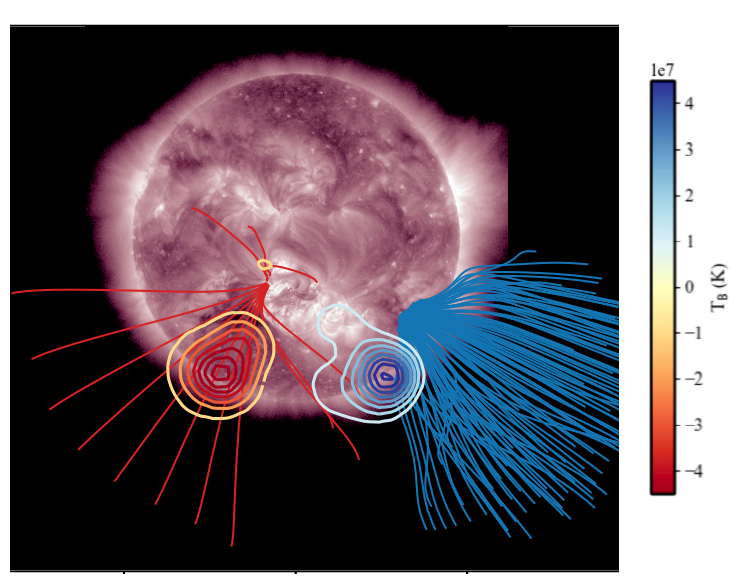

Fig. 9. Polarised moving radio bursts and open magnetic field regions. Radio contours and extrapolated open magnetic field lines are overlaid on AIA $211 \AA$ images at 13:50 UT. The positive open field lines (blue) and left-handed circularly polarised burst (blue contours) are located to the right of the active region where the CME originates, while the negative open field lines (red) and right-handed circularly polarised burst (red contours) are located to the left of the active region. The sense of polarisation is from the point of view of the observer. The two radio sources have an opposite sense of circular polarisation since they propagate on either sides of the active region, through open field regions also of opposite polarity.

enough to drive a shock at this time (the lateral CME speed is $<500 \mathrm{~km} \mathrm{~s}^{-1}$ before 13:50 UT when Source 1 occurs). Source 1 is also less polarised than Sources 2 and 3 and has less steep spectral indices that fall in the range of gyro-synchrotron spectral indices (Nita et al. 2004). However, this emission appears narrowband in the NRH images and, given the lack of imaging at lower frequencies, it is not possible to draw other conclusions on the nature of this emission. Another possibility is that Source 1, which occurs at an earlier time, has access to differently oriented closed magnetic field lines compared to Source 3, to explain its sense of circular polarisation.

In the case of Sources 2 and 3, we provided evidence that they are emitted by plasma radiation that moves outwards with the CME flanks. In the case that scattering is not significant at $\mathrm{NRH}$ frequencies, the emission is predominantly located outside the reconstructed CME shell. At this time, we also showed that a faint coronal wave is present on the western hemisphere and that the CME becomes fast enough to drive a low coronal shock. A CME-driven shock is a likely possibility for the acceleration of energetic electrons producing radio emission outside the CME. In the event that scattering is indeed significant at the NRH frequencies, then these radio sources, in particular Source 3 would be located inside the reconstructed CME shell. In this case, the radio emission would represent plasma radiation inside the CME legs and the origin of accelerated electrons may be a reconnecting current sheet behind the CME (Démoulin et al. 2012; Morosan et al. 2019b). Thus, the radio sources would also move outwards along a constant density gradient with the CME, however they would not be located at the flank but inside the CME behind its flanks. In either case, the radio source propagation is in the direction of the CME lateral expansion and the moving radio sources are closely related to the propagation of the $\mathrm{CME}$ flanks through the low corona. 


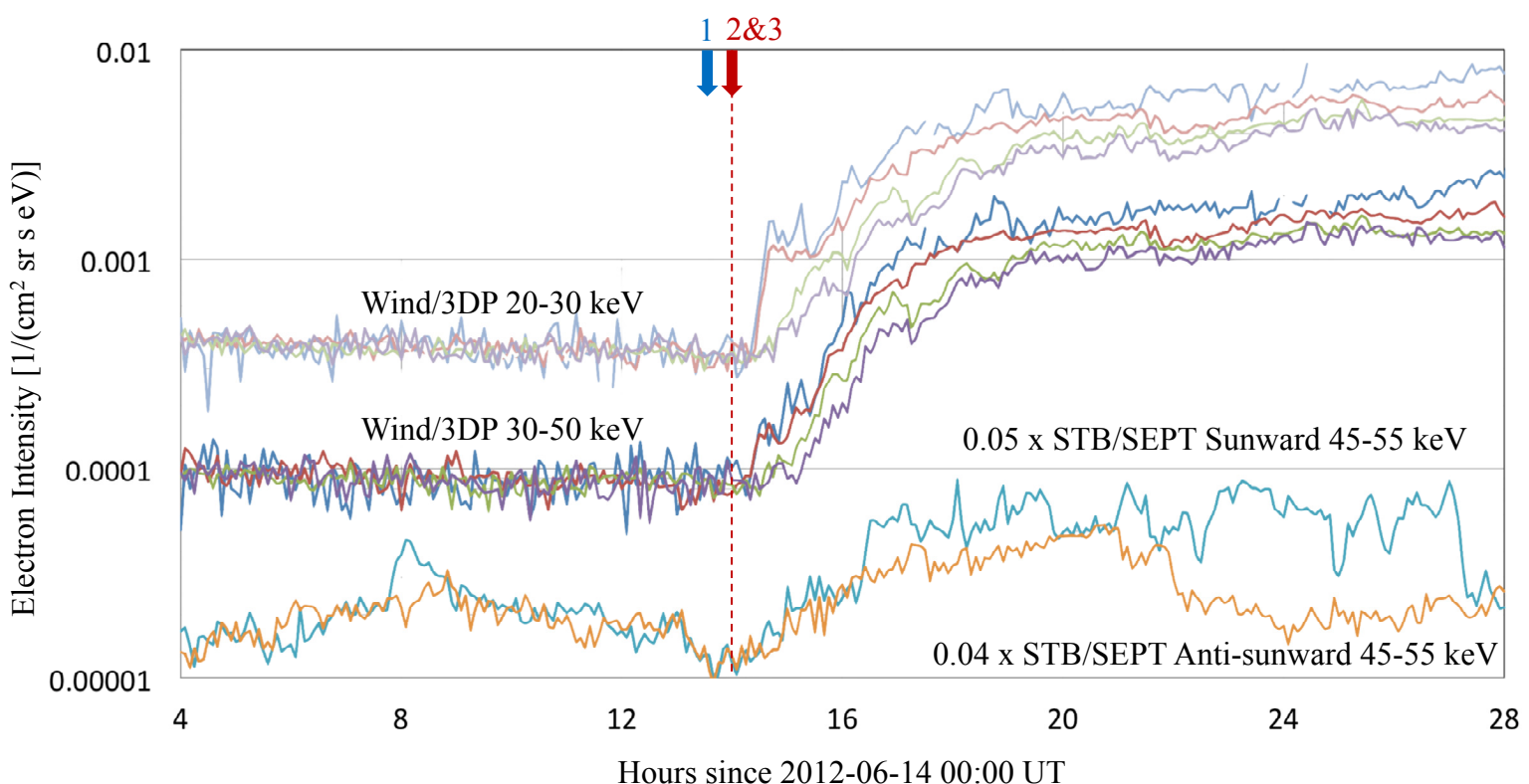

Fig. 10. Energetic electrons observed in situ at $1 \mathrm{AU}$. The electrons were observed by two spacecraft: STEREO-B/SEPT at $45-55 \mathrm{keV}$ and WIND/3DP at 20-30 and 30-50 keV. The start time of Sources 1, 2, and 3 is annotated with the blue and red arrows. The STEREO-B electron intensities were multiplied by factors of 0.05 for the sunward-looking telescope and by 0.04 for anti-sunward-looking telescopes and the data were normalised so that the intensities agree at 16:00 and 20:00 UT. The electron intensities observed by the two spacecraft started increasing shortly after the onset of Sources 2 and 3, denoted by the vertical red dashed line.

The availability of open magnetic field lines and regions of high density plasma inside the coronal streamers surrounding the CME appears to facilitate the acceleration of electrons at the CME flanks. The presence of open field lines can also facilitate the escape of energetic electrons to interplanetary space. Energetic electrons associated with this CME have indeed been observed in situ at $1 \mathrm{AU}$ (astronomical unit, where $\left.1 \mathrm{AU}=1.5 \times 10^{11} \mathrm{~m}\right)$ by the Solar Electron and Proton Telescope (SEPT; Luhmann et al. 2008) on board STEREO-B and the Three-Dimensional Plasma (3DP; Lin et al. 1995) instrument on board Wind (Ogilvie \& Desch 1997) located near Earth. Both spacecraft observed an increase in electron intensities shortly after the onset of Sources 2 and 3 (Fig. 10). The electrons observed by Wind in two energy channels (lighter shades for $20-30 \mathrm{keV}$ and darker shades for $30-50 \mathrm{keV}$ in Fig. 10) have pitch angles of $11^{\circ}$ (blue), $56^{\circ}$ (red), $101^{\circ}$ (green), and $146^{\circ}$ (purple), indicating that they are travelling from a sunward direction. The near-relativistic electrons observed by STEREO-B are plotted from the sunward (blue) and anti-sunward (orange) directions and have energies of 45-55 keV in Fig. 10.

The presence of in-situ electrons indicates a low-coronal acceleration region co-temporal with the appearance of Sources 2 and 3. Assuming a travel path of $1.2 \mathrm{AU}$ for the electrons, and a time of $8 \mathrm{~min}$ for the radio emission to travel from the Sun to Earth, the delay times of the energetic electrons with respect to Sources 2 and 3 are the following: $15.9 \mathrm{~min}$ at $50 \mathrm{keV}$, $22.1 \mathrm{~min}$ at $30 \mathrm{keV}$, and $28.4 \mathrm{~min}$ at $20 \mathrm{keV}$. Accounting for these delay times, the acceleration of the in-situ electrons in the corona occurs shortly or only a few minutes after the onset time of Sources 2 and 3. Both radio sources and energetic electrons start at a later stage in the eruption and not during the impulsive energy release of the flare. In the case of in-situ electrons, this indicates the presence of a low-coronal shock wave accelerating these particles (Pomoell et al. 2011). Sources 2 and 3 are short-lived in comparison to the electron event; however, as the CME continues to propagate further away from the Sun, any possible radio emission would move outside the NRH fre- quency band towards lower and lower frequencies. The coincidental onset time of the in-situ electrons and Sources 2 and 3 may indicate that both events originated at the CME flanks, and a CME shock may be the origin of the observed in-situ electrons. In addition, Sources 2 and 3 occurred in regions where the CME had ample access to open magnetic field lines, where electrons could escape along the interplanetary magnetic field. The regions where Sources 2 and 3 originate may therefore be related to the origin of the observed in-situ energetic electrons.

\section{Conclusions}

So far, moving radio bursts have been classified either as emission due to electrons trapped inside the CME or electrons accelerated by magnetic reconnection processes outside the CME in the case of type IVs. However, moving radio bursts coincident with type IV emission and CME shocks have not been classified before, unless they show clear type II or herringbone structures. Here, we have shown a new origin of moving radio sources, other than type IIs or herringbones, that are generated at the CME flanks. Some of the moving bursts are likely associated with electrons accelerated by the CME-driven shock or electrons accelerated inside the CME also moving outwards with the CME flanks. The moving radio bursts in this study behave similarly to type II radio bursts or herringbones that propagate outwards in the solar corona (Carley et al. 2013; Zucca et al. 2018; Morosan et al. 2019a). Simulations of type II radio bursts, emitted during CMEs with strong lateral expansions such as the one observed here, can produce broad fundamental and harmonic type II lanes in dynamic spectra, that are indistinguishable from each other (Knock \& Cairns 2005). This could be the case in our observations, where we do not observe any type II emission lanes in dynamic spectra but instead a broader continuum emission that resembles a type IV with superimposed fine structures.

In our study, plane-of-sky ground observations were not sufficient to identify the nature of the moving radio emission observed. The additional perspectives provided by the STEREO 
mission were invaluable for this study. Future observations of moving radio bursts will benefit from better imaging capabilities at multiple frequencies combined with high-resolution spectroscopy, to be able to distinguish the type of radio emission observed. Such observations are now possible with the Low Frequency Array (LOFAR) or the Murchinson Widefield Array (MWA). These observations, combined with additional vantage points from the STEREO-A spacecraft and future L5 missions, can provide the opportunity to understand the 3D nature and propagation of CMEs and associated radio emission.

Acknowledgements. The results presented here have been achieved under the framework of the Finnish Centre of Excellence in Research of Sustainable Space (Academy of Finland Grant numbers 312390, 312357, and 312351), which we gratefully acknowledge. E. P. acknowledges the Doctoral Programme in Particle Physics and Universe Sciences (PAPU) at the University of Helsinki. R. V. acknowledges the financial support of the Academy of Finland (project 309939). M. P. acknowledges the European Research Council (ERC) Consolidator grant 682068-PRESTISSIMO, and Academy of Finland Grants 312351 and 309937. E. K. J. K. acknowledges the ERC under the European Union's Horizon 2020 Research and Innovation Programme Project SolMAG 724391, and Academy of Finland Project 310445. We would like to acknowledge the Nançay Radioheliograph, funded by the French Ministry of Education and the Région Centre in France, the Nançay Decametric Array hosted at the Nançay Radio Observatory/Unité Scientifique de Nançay of the Observatoire de Paris (USR 704-CNRS, supported by Université de Orléans, OSUC and the Région Centre) and the e-Callisto Birr spectrometer located at the Rosse Observatory and supported by Trinity College Dublin. We thank the Radio Solar Database service at LESIA \& USN (Observatoire de Paris) for making the NRH/NDA data available. We would like to thank the Wind/3DP and STEREO/SEPT instrument teams for making their data publicly available.

\section{References}

Aschwanden, M. J. 2009, Ann. Geophys., 27, 3275

Aulanier, G., Török, T., Démoulin, P., \& DeLuca, E. E. 2010, ApJ, 708, 314

Bastian, T. S., Benz, A. O., \& Gary, D. E. 1998, ARA\&A, 36, 131

Benz, A. O., \& Tarnstrom, G. L. 1976, ApJ, 204, 597

Boischot, A., \& Clavelier, B. 1968, in Structure and Development of Solar Active Regions, ed. K. O. Kiepenheuer, IAU Symp., 35, 565

Boischot, A., Rosolen, C., Aubier, M. G., et al. 1980, Icarus, 43, 399

Brueckner, G. E., Howard, R. A., Koomen, M. J., et al. 1995, Sol. Phys., 162, 357

Cairns, I. H., \& Robinson, R. D. 1987, Sol. Phys., 111, 365

Cane, H. V., \& White, S. M. 1989, Sol. Phys., 120, 137

Carley, E. P., Long, D. M., Byrne, J. P., et al. 2013, Nat. Phys., 9, 811

Carley, E. P., Vilmer, N., Simões, P. J. A., \& Ó Fearraigh, B. 2017, A\&A, 608, A137

Chen, P. F. 2011, Sol. Phys., 8, 1

Chen, J. 2017, Phys. Plasmas, 24, 090501

Chen, Y., Song, H. Q., Li, B., et al. 2010, ApJ, 714, 644

Chiuderi Drago, F., Landi, E., Fludra, A., \& Kerdraon, A. 1999, A\&A, 348, 261

Démoulin, P., Vourlidas, A., Pick, M., \& Bouteille, A. 2012, ApJ, 750, 147

Dere, K. P., Brueckner, G. E., Howard, R. A., Michels, D. J., \& Delaboudiniere, J. P. 1999, ApJ, 516, 465

Domingo, V., Fleck, B., \& Poland, A. I. 1995, Sol. Phys., 162, 1

Downs, C., Roussev, I. I., van der Holst, B., Lugaz, N., \& Sokolov, I. V. 2012, ApJ, 750, 134

Dulk, G. A. 1973, Sol. Phys., 32, 491

Dulk, G. A., \& Suzuki, S. 1980, A\&A, 88, 203

Dulk, G. A., Jacques, S., Smerd, S. F., et al. 1976, Sol. Phys., 49, 369

Evans, R. M., Opher, M., Manchester, IV, W. B., \& Gombosi, T. I. 2008, ApJ, 687,1355

Feng, S. W., Chen, Y., Kong, X. L., et al. 2012, ApJ, 753, 21

Forbes, T. G. 2000, J. Geophys. Res. Space Phys., 105, 23153

Gallagher, P. T., Lawrence, G. R., \& Dennis, B. R. 2003, ApJ, 588, L53

Gary, D. E., Dulk, G. A., House, L. L., et al. 1985, A\&A, 152, 42
Grechnev, V. V., Uralov, A. M., Chertok, I. M., et al. 2011, Sol. Phys., 273, 433 Green, L. M., Török, T., Vršnak, B., Manchester, W., \& Veronig, A. 2018, Space Sci. Rev., 214, 46

Holman, G. D., \& Pesses, M. E. 1983, ApJ, 267, 837

Howard, R. A., Moses, J. D., Vourlidas, A., et al. 2008, Space Sci. Rev., 136, 67 Illing, R. M. E., \& Hundhausen, A. J. 1985, J. Geophys. Res. Space Phys., 90, 275

James, A. W., Green, L. M., Palmerio, E., et al. 2017, Sol. Phys., 292, 71

Kai, K. 1970, Sol. Phys., 11, 456

Kai, K. 1978, Sol. Phys., 56, 417

Kaiser, M. L., Kucera, T. A., Davila, J. M., et al. 2008, Space Sci. Rev., 136, 5

Kerdraon, A., \& Delouis, J. M. 1997, in Coronal Physics from Radio and Space

Observations, ed. G. Trottet (Berlin: Springer Verlag), Lect. Notes Phys., 483, 192

Klassen, A., Bothmer, V., Mann, G., et al. 2002, A\&A, 385, 1078

Knock, S. A., \& Cairns, I. H. 2005, J. Geophys. Res. Space Phys., 110, A01101

Kozarev, K. A., Korreck, K. E., Lobzin, V. V., Weber, M. A., \& Schwadron, N. A. 2011, ApJ, 733, L25

Lemen, J. R., Title, A. M., Akin, D. J., et al. 2012, Sol. Phys., 275, 17

Lin, R. P., Anderson, K. A., Ashford, S., et al. 1995, Space Sci. Rev., 71, 125

Long, D. M., Gallagher, P. T., McAteer, R. T. J., \& Bloomfield, D. S. 2008, ApJ, 680, L81

Luhmann, J. G., Curtis, D. W., Schroeder, P., et al. 2008, Space Sci. Rev., 136, 117

Mann, G., Klassen, A., Classen, H. T., et al. 1996, A\&AS, 119, 489

McCauley, P. I., Cairns, I. H., \& Morgan, J. 2018, Sol. Phys., 293, 132

McCauley, P. I., Cairns, I. H., White, S. M., et al. 2019, Sol. Phys., 294, 106

Melrose, D. B. 2009, in Universal Heliophysical Processes, eds. N. Gopalswamy, \& D. F. Webb, IAU Symp., 257, 305

Melrose, D. B., \& Sy, W. N. 1972, Aust. J. Phys., 25, 387

Morosan, D. E., Carley, E. P., Hayes, L. A., et al. 2019a, Nat. Astron., 3, 452

Morosan, D. E., Kilpua, E. K. J., Carley, E. P., \& Monstein, C. 2019b, A\&A, 623, A63

Morosan, D. E., Palmerio, E., Lynch, B. J., \& Kilpua, E. K. J. 2020, A\&A, 633, A141

Nelson, G. J., \& Melrose, D. B. 1985, in Solar Radiophysics: Studies of Emission from the Sun at Metre Wavelengths (A87-13851 03-92), eds. D. J. McLean, \& N. R. Labrum (Cambridge and New York: Cambridge University Press), 333

Newkirk, G. J. 1961, ApJ, 133, 983

Nita, G. M., Gary, D. E., \& Lee, J. 2004, ApJ, 605, 528

Oakes, M. E., Michie, R. B., Tsui, K. H., \& Copeland, J. E. 1979, J. Plasma Phys., 21, 205

Ogilvie, K. W., \& Desch, M. D. 1997, Adv. Space Res., 20, 559

Palmerio, E., Kilpua, E. K. J., James, A. W., et al. 2017, Sol. Phys., 292, 39

Pesnell, W. D., Thompson, B. J., \& Chamberlin, P. C. 2012, Sol. Phys., 275, 3

Pomoell, J., Vainio, R., \& Kissmann, R. 2011, Astrophys. Space Sci. Trans., 7, 387

Poquerusse, M., Steinberg, J. L., Caroubalos, C., Dulk, G. A., \& MacQueen, R. M. 1988, A\&A, 192, 323

Robinson, R. D. 1978, ApJ, 222, 696

Robinson, R. D. 1983, PASA, 5, 208

Saito, K., Poland, A. I., \& Munro, R. H. 1977, Sol. Phys., 55, 121

Schrijver, C. J., \& De Rosa, M. L. 2003, Sol. Phys., 212, 165

Smerd, S. F., \& Dulk, G. A. 1971, in Solar Magnetic Fields, ed. R. Howard, IAU Symp., 43, 616

Stewart, R. T. 1971, Aust. J. Phys., 24, 209

Stewart, R. T., Howard, R. A., Hansen, F., Gergely, T., \& Kundu, M. 1974, Sol. Phys., 36, 219

Suzuki, S., Stewart, R. T., \& Magun, A. 1980, in Radio Physics of the Sun, eds. M. R. Kundu, \& T. E. Gergely, IAU Symp., 86, 241

Thernisien, A. F. R., Howard, R. A., \& Vourlidas, A. 2006, ApJ, 652, 763

Thernisien, A., Vourlidas, A., \& Howard, R. A. 2009, Sol. Phys., 256, 111

Vourlidas, A., Wu, S. T., Wang, A. H., Subramanian, P., \& Howard, R. A. 2003, ApJ, 598, 1392

Vourlidas, A., Lynch, B. J., Howard, R. A., \& Li, Y. 2013, Sol. Phys., 284, 179

Vršnak, B., Klein, K.-L., Warmuth, A., Otruba, W., \& Skender, M. 2003, Sol. Phys., 214, 325

Wang, W., Zhu, C., Qiu, J., et al. 2019, ApJ, 871, 25

Zucca, P., Carley, E. P., McCauley, J., et al. 2012, Sol. Phys., 280, 591

Zucca, P., Morosan, D. E., Rouillard, A. P., et al. 2018, A\&A, 615, A89 